\title{
Chaos Synchronization of Financial Chaotic System with External Perturbation
}

\author{
Chengrong Xie, ${ }^{1,2}$ Yuhua $\mathrm{Xu},{ }^{1,2}$ and Dongbing Tong ${ }^{3}$ \\ ${ }^{1}$ Department of Mathematics and Finance, Yunyang Teachers' College, Hubei, Shiyan 442000, China \\ ${ }^{2}$ School of Finance, Nanjing Audit University, Jiangsu 211815, China \\ ${ }^{3}$ College of Electronic and Electrical Engineering, Shanghai University of Engineering Science, Shanghai 201620, China
}

Correspondence should be addressed to Yuhua Xu; yuhuaxu2004@163.com

Received 8 September 2014; Revised 25 December 2014; Accepted 25 December 2014

Academic Editor: Qingling Zhang

Copyright (c) 2015 Chengrong Xie et al. This is an open access article distributed under the Creative Commons Attribution License, which permits unrestricted use, distribution, and reproduction in any medium, provided the original work is properly cited.

\begin{abstract}
This paper investigates the problem of two kinds of function projective synchronization of financial chaotic system with definite integration scaling function, which are not fully considered in the existing research. Different from the previous methods, in this paper, the following two questions are investigated: (1) two kinds of the definite integration scaling function projective synchronization are given; (2) the upper and lower limit of the definite integral scaling function are the bound dynamical systems. Finally, illustrative example is provided to show the effectiveness of this method.
\end{abstract}

\section{Introduction}

In nonlinear areas, researchers are striving to utilize the theory of nonlinear dynamics, especially the chaos theory, to study the complexity of economic and financial systems in recent years [1-6]. Since Strotz et al. have done the pioneering work in this area [7], various economics chaotic models have been proposed, such as the Kaldorian model [8], the IS-LM model [9-11], the hyperchaotic finance system [12], and other nonlinear dynamical models [13-16].

It is well known that economic chaotic systems are inevitably influenced by external disturbances stemmed from environmental interference [17-22], and external disturbances may lead to the destabilization of economic and financial chaotic systems and cause undesirable results. It is necessary to study the global stabilization of economic and financial chaotic systems under the presence of external disturbance. Some results have been reported about stabilization of complex systems with external disturbance [23, 24].

In the past few decades, projective synchronization of chaotic systems has attracted a great deal of attention, because projective synchronization can obtain the effective results faster. Later, with the deepening of the research, various projective synchronization methods were discussed.
For example, in [25-29], the authors investigated function projective synchronization of chaotic systems, and the scaling function is selected to be constant or unity. In [30-33], the authors discussed function projective synchronization of chaotic systems with the unary scaling function. Up to now, most of research efforts mentioned above have concentrated on studying the presetting scaling function in numerical examples yet. About the problem of the definite integral scaling function, to the best of the authors' knowledge, it has rarely been investigated, which still remains open. Motivated by the existing works, in the present paper, we intend to derive some scaling function projective synchronization criteria for the chaotic financial system. Different from the previous methods, our main contributions are as follows: (1) we give two kinds of the new scaling function projective synchronization, that is, the definite integration scaling function projective synchronization and adaptive definite integration scaling function projective synchronization; (2) we consider that the upper and lower limit of the definite integral scaling function are the bound dynamical systems, which can be the stable equilibrium point, the stable periodic orbit, or the chaotic attractor.

The rest of this paper is organized as follows: Model and preliminaries are presented in Section 2. The sufficient 
conditions of synchronization are given in Section 3. Section 4 presents an example and relates simulation results. The conclusions are given in Section 5.

\section{Model and Preliminaries}

In 2001, a new dynamical model of the financial system was proposed. The model is described by the following threedimensional system:

$$
\begin{aligned}
& \dot{x}_{1}=x_{3}+\left(x_{2}-a\right) x_{1}, \\
& \dot{x}_{2}=1-b x_{2}-x_{1}^{2}, \\
& \dot{x}_{3}=-x_{1}-c x_{3},
\end{aligned}
$$

where $x_{1}$ is the interest rate, $x_{2}$ is the investment demand, and $x_{3}$ is the price index. $a>0$ represents the saving amount, $b>0$ represents the cost per investment, and $c>0$ represents the elasticity of demand of commercial markets. When parameters $a=0.9, b=0.2$, and $c=1.5$, system (1) exhibits chaotic behavior [14].

Now, we consider the following system with external perturbation and system (1) changes into the following system:

$$
\begin{aligned}
& \dot{y}_{1}=y_{3}+\left(y_{2}-a_{2}\right) y_{1}, \\
& \dot{y}_{2}=1-b_{2} y_{2}-y_{1}^{2}, \\
& \dot{y}_{3}=-y_{1}-c_{2} y_{3}+h v,
\end{aligned}
$$

where $\dot{v}=-1.2 y_{1} y_{2}-0.5 v$.

In essence, three-dimensional system (2) with external perturbation can be seen as the following four-dimensional system without external perturbation:

$$
\begin{aligned}
& \dot{y}_{1}=y_{3}+\left(y_{2}-a_{2}\right) y_{1}, \\
& \dot{y}_{2}=1-b_{2} y_{2}-y_{1}^{2}, \\
& \dot{y}_{3}=-y_{1}-c_{2} y_{3}+h v, \\
& \dot{v}=-1.2 y_{1} y_{2}-0.5 v,
\end{aligned}
$$

and system (3) exhibits chaotic behavior for the parameters $a=0.9, b=0.2, c=1.5$, and $h=-5$ (see Figures $1-6$ ).

The bifurcation diagram would be far better to summarize all of the possible behaviors as the parameter varies on one diagram. For $-14 \leq h \leq 1.5$, the bifurcation diagram of fourdimensional system (3) shows the complicated bifurcation phenomena in Figure 7.

Remark 1. The above analysis shows that the chaotic financial system remained as chaotic characteristics in the appropriate external perturbation. More detailed analysis on chaotic system (2) will be reported in a forthcoming paper.

\section{Main Results}

In this section, we give two kinds of integral proportional function synchronization definition and discuss the synchronization of the chaotic finance system.

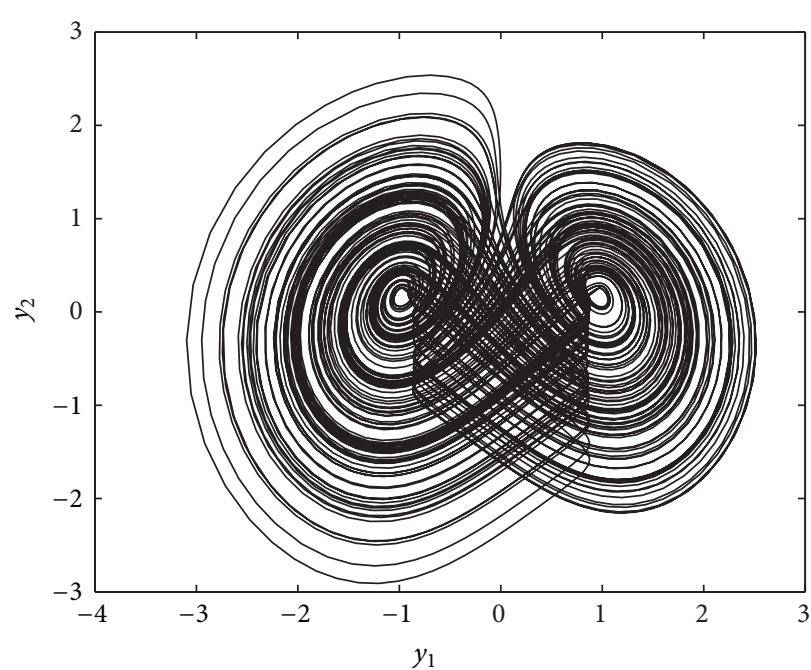

FIgURE 1: $y_{1}-y_{2}$ phase plane, the chaotic attractor.

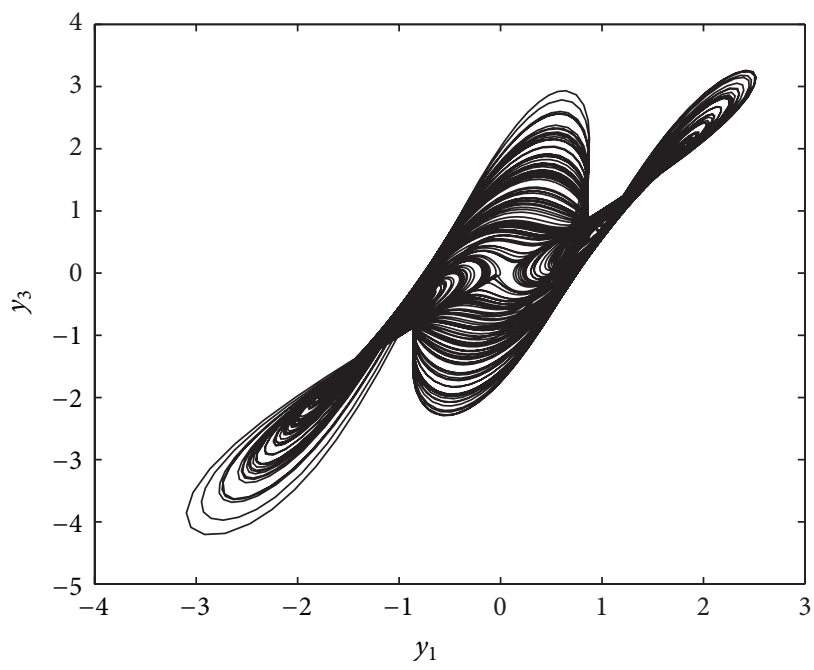

FIGURE 2: $y_{1}-y_{3}$ phase plane, the chaotic attractor.

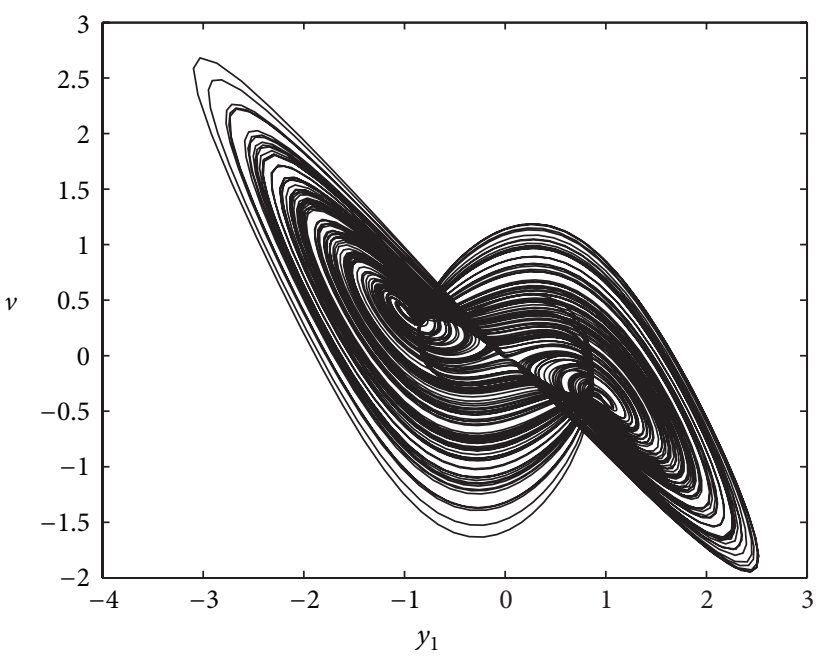

FIGURE 3: $y_{1}-v$ phase plane, the chaotic attractor. 


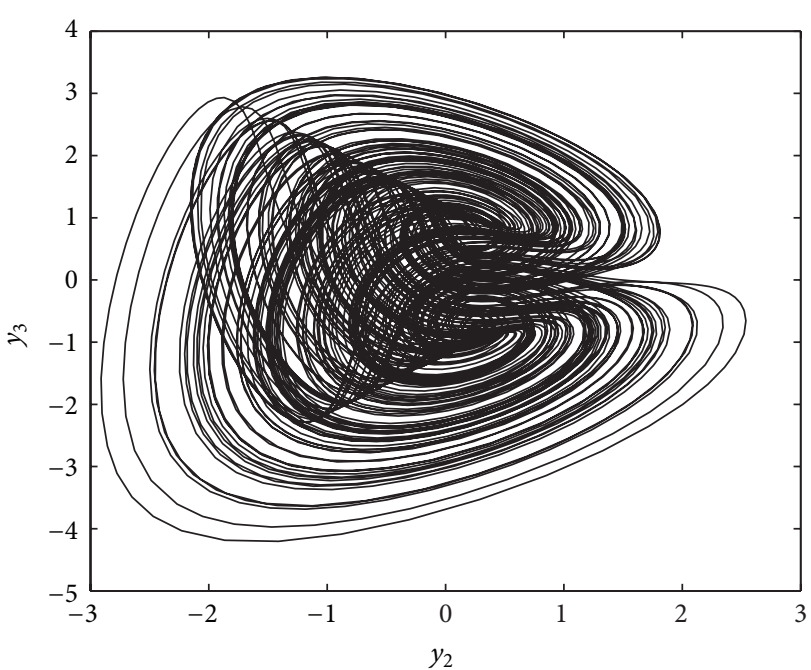

FIGURE 4: $y_{2}-y_{3}$ phase plane, the chaotic attractor.

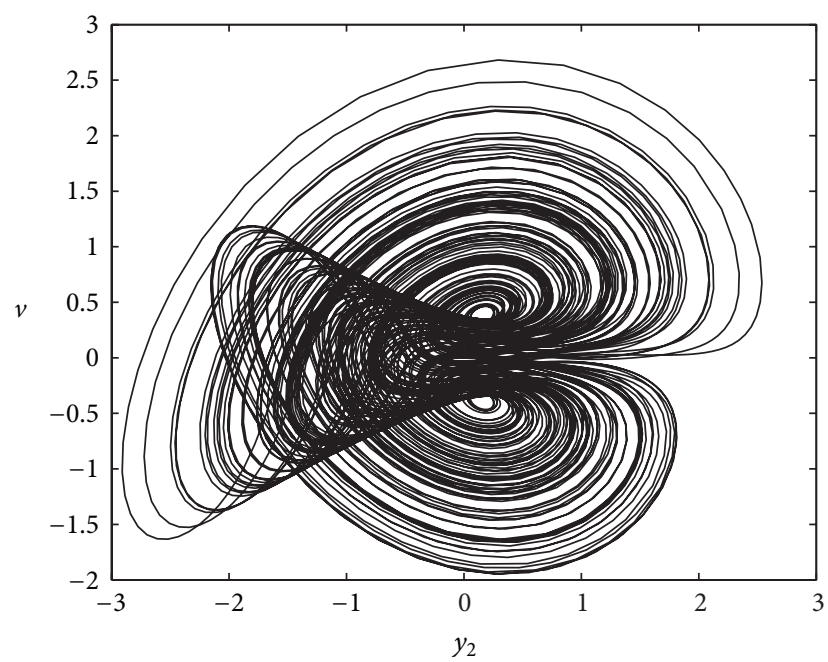

FIGURE 5: $y_{2}-v$ phase plane, the chaotic attractor.

The drive system is given by system (1), and the response system with external perturbation is the following controlled system:

$$
\begin{aligned}
& \dot{y}_{1}=y_{3}+\left(y_{2}-a\right) y_{1}+u_{1} \\
& \dot{y}_{2}=1-b y_{2}-y_{1}^{2}+u_{2} \\
& \dot{y}_{3}=-y_{1}-c y_{3}+h v+u_{3},
\end{aligned}
$$

where $\dot{v}=-1.2 y_{1} y_{2}-0.5 v$.

Definition 2. For systems (1) and (4), if

$$
\lim _{t \rightarrow \infty}\left\|e_{i}\right\|=\lim _{t \rightarrow \infty}\left\|y_{i}-\left(\int_{\delta_{i}}^{w_{i}} s d s\right) x_{i}\right\|=0, \quad i=1,2, \ldots, n,
$$

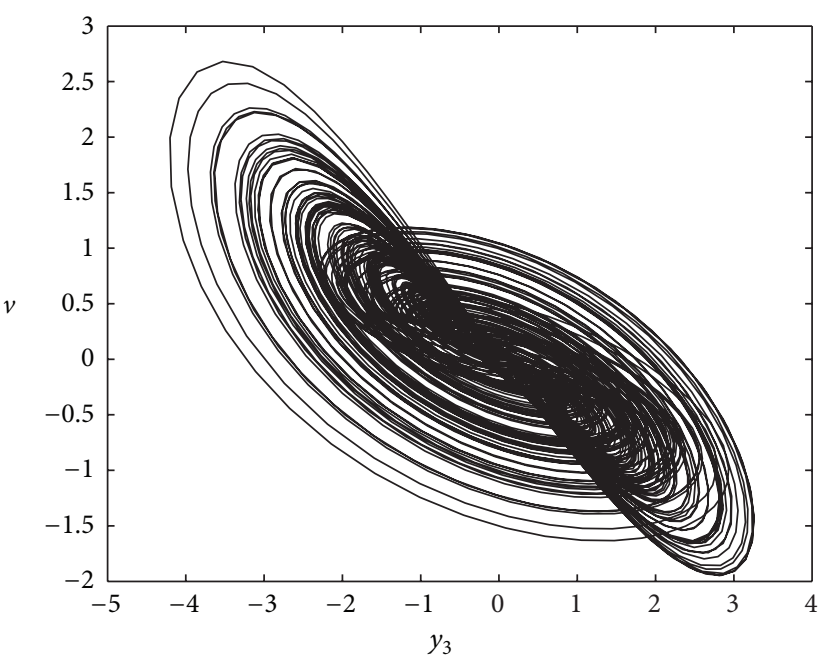

FIGURE 6: $y_{3}-v$ phase plane, the chaotic attractor.

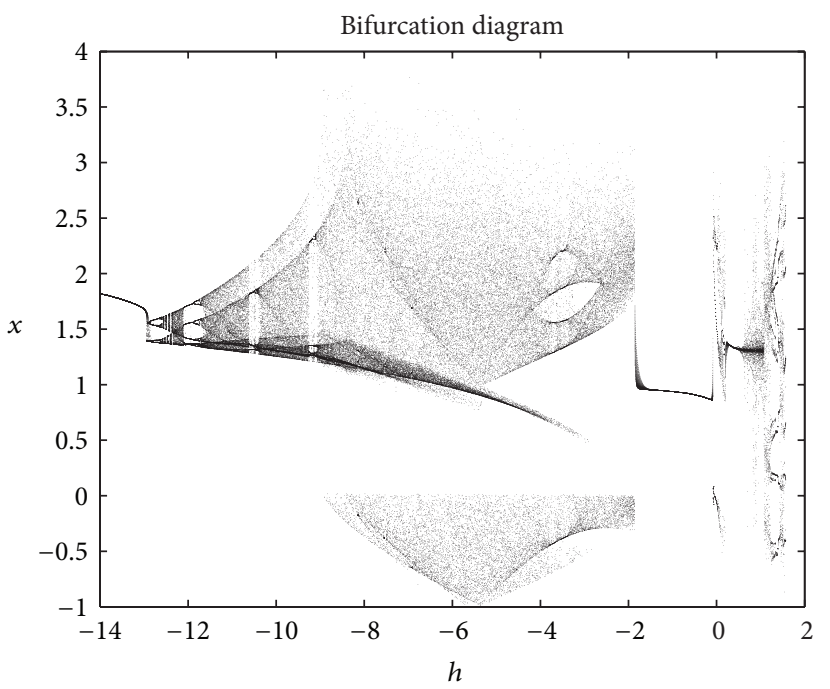

FIGURE 7: Bifurcation diagram of system (3).

then the status of the response system and the drive system is called adaptive definite integration scaling function projective synchronization (ADISFPS), where $\dot{w}_{i}=f\left(w_{i}, t\right)$ and $\dot{\delta}_{i}=g\left(\delta_{i}, t\right)$ are bounded dynamical systems.

Remark 3. For Definition 2, when the upper and lower limit of integration of the scaling function are boundless, one problem should be taken into account if $e_{i}=y_{i}-\left(\int_{\delta_{i}}^{w_{i}} s d s\right) x_{i}$ is equal to infinite value. To achieve synchronization, that is, $e_{i}=y_{i}-\left(\int_{\delta_{i}}^{w_{i}} s d s\right) x_{i}$ is equal to 0 in the process of synchronization, we let the upper and lower limit of integration of the scaling function have the boundary; thus, we assume that for the dynamical systems $\dot{w}_{i}=f\left(w_{i}, t\right)$ and $\dot{\delta}_{i}=g\left(\delta_{i}, t\right)$ there exists the stable equilibrium point, the stable periodic orbit, or the chaotic attractor in the phase space, which satisfies the assumption. 
Remark 4. Synchronization of the financial systems shows they can maintain consistency; that is, the economic systems in two different areas are to keep synchronized development by applying the appropriate control conditions. In economic activities, the driving system can be understood as a virtual economic goal, the response system is thought as a controlled object, and the manager's goal is to control a controlled object to a virtual economic goal. In practice, due to the complexity of the economic environment, the financial systems are not always completely synchronization; some complex synchronization methods should be considered. Therefore, the study of projective synchronization is meaningful in real economic activities.

Theorem 5. System (1) and system (4) are asymptotically synchronized with the following adaptive control mechanism:

$$
\begin{gathered}
u_{1}=\left(w_{1} \dot{w}_{1}-\delta_{1} \dot{\delta}_{1}\right) x_{1}-y_{1} y_{2}+\left(\int_{\delta_{1}}^{w_{1}} s d s\right) x_{1} x_{2}+k_{1} e_{1} \\
u_{2}=\left(w_{2} \dot{w}_{2}-\delta_{2} \dot{\delta}_{2}\right) x_{2} \\
+\int_{\delta_{2}}^{w_{2}} s d s-1+y_{1}^{2}-\left(\int_{\delta_{2}}^{w_{2}} s d s\right) x_{1}^{2}+k_{2} e_{2} \\
u_{3}=\left(w_{3} \dot{w}_{3}-\delta_{3} \dot{\delta}_{3}\right) x_{3}-h v+k_{3} e_{3} \\
\dot{v}=-1.2 y_{1} y_{2}-0.5 v \\
\dot{w}_{i}=f\left(w_{i}, t\right), \quad i=1,2,3 \\
\dot{\delta}_{i}=g\left(\delta_{i}, t\right), \quad i=1,2,3 \\
\dot{k}_{i}=-\lambda_{i} e_{i}^{2}, \quad i=1,2,3 .
\end{gathered}
$$

Proof. The error system between driving system (1) and response system (4) can be expressed by

$$
\begin{aligned}
\dot{e}_{1}= & y_{3}+\left(y_{2}-a_{2}\right) y_{1}+u_{1}-\left(\int_{\delta_{1}}^{w_{1}} s d s\right)\left(x_{3}+\left(x_{2}-a\right) x_{1}\right) \\
& -\left(w_{1} \dot{w}_{1}-\delta_{1} \dot{\delta}_{1}\right) x_{1}, \\
\dot{e}_{2}= & 1-b_{2} y_{2}-y_{1}^{2}+u_{2}-\left(\int_{\delta_{2}}^{w_{2}} s d s\right)\left(1-b x_{2}-x_{1}^{2}\right) \\
& -\left(w_{2} \dot{w}_{2}-\delta_{2} \dot{\delta}_{2}\right) x_{2}, \\
\dot{e}_{3}= & -y_{1}-c_{2} y_{3}+h v+u_{3}-\left(\int_{\delta_{3}}^{w_{3}} s d s\right)\left(-x_{1}-c x_{3}\right) \\
& -\left(w_{3} \dot{w}_{3}-\delta_{3} \dot{\delta}_{3}\right) x_{3} .
\end{aligned}
$$

Substituting (6) into (11) yields

$$
\begin{aligned}
& \dot{e}_{1}=e_{3}-a e_{1}+k_{1} e_{1}, \\
& \dot{e}_{2}=-b e_{2}+k_{2} e_{2}, \\
& \dot{e}_{3}=-e_{1}-c e_{3}+k_{3} e_{3} .
\end{aligned}
$$

Choose a candidate Lyapunov function as follows:

$$
V=\frac{1}{2}\left(e_{1}^{2}+e_{2}^{2}+e_{3}^{2}\right)+\frac{1}{2} \sum_{i=1}^{3} \frac{1}{\lambda_{i}}\left(k_{i}+L\right)^{2},
$$

where $L>\max \{-a,-b,-c\}$.

Then the differentiation of $V$ along the trajectories of (12) is

$$
\begin{aligned}
\dot{V}= & e_{1} \dot{e}_{1}+e_{2} \dot{e}_{2}+e_{3} \dot{e}_{3}+\sum_{i=1}^{3} \frac{1}{\lambda_{i}}\left(k_{i}+L\right) \dot{k}_{i} \\
= & e_{1}\left(e_{3}-a e_{1}+k e_{1}\right)+e_{2}\left(-b e_{2}+k e_{2}\right) \\
& +e_{3}\left(-e_{1}-c e_{3}+k e_{3}\right)-\sum_{i=1}^{3}\left(k_{i}+L\right) e_{i}^{2} \\
= & e_{1}\left(e_{3}-a e_{1}\right)-b e_{2}^{2}+e_{3}\left(-e_{1}-c e_{3}\right)-\sum_{i=1}^{3} L e_{i}^{2} \\
= & -(L+a) e_{1}^{2}-(L+b) e_{2}^{2}-(L+c) e_{3}^{2}<0 .
\end{aligned}
$$

It is clear that $V$ is positive definite and $\dot{V}$ is negative definite. According to the Lyapunov stability theorem, the trivial solution of error system (11) is asymptotically stable, which implies that the synchronization of systems (1) and (4) is achieved. The proof of the theorem is completed.

Remark 6. Synchronization conditions of the chaotic financial system with external perturbation are derived from Theorem 5. When the chaotic financial system with external perturbation is thought as the four-dimensional system without external perturbation, similar to Theorem 5, we may get the following synchronization condition.

Remark 7. In [25-33], research efforts have concentrated on studying a presetting scaling function with numerical examples. In this paper, the scaling function can be the definite integration, and the upper and lower limit of the definite integral scaling function are the bound dynamical systems.

The drive system is given by system (1), and the response system without external perturbation is the following controlled system:

$$
\begin{aligned}
& \dot{y}_{1}=y_{3}+\left(y_{2}-a_{2}\right) y_{1}+u_{1}, \\
& \dot{y}_{2}=1-b_{2} y_{2}-y_{1}^{2}+u_{2}, \\
& \dot{y}_{3}=-y_{1}-c_{2} y_{3}+h v+u_{3}, \\
& \dot{v}=-1.2 y_{1} y_{2}-0.5 v+u_{4} .
\end{aligned}
$$


Theorem 8. System (1) and system (15) are asymptotically synchronized with the following adaptive control mechanism:

$$
\begin{gathered}
u_{1}=\left(w_{1} \dot{w}_{1}-\delta_{1} \dot{\delta}_{1}\right) x_{1}-y_{1} y_{2}+\left(\int_{\delta_{1}}^{w_{1}} s d s\right) x_{1} x_{2}+k_{1} e_{1}, \\
u_{2}=\left(w_{2} \dot{w}_{2}-\delta_{2} \dot{\delta}_{2}\right) x_{2}+\int_{\delta_{2}}^{w_{2}} s d s \\
-1+y_{1}^{2}-\left(\int_{\delta_{2}}^{w_{2}} s d s\right) x_{1}^{2}+k_{2} e_{2}, \\
u_{4}=\left(w_{4} \dot{w}_{4}-\delta_{4} \dot{\delta}_{4}\right) x_{4}+1.2 y_{1} y_{2}+0.5 v+k_{4} e_{4}, \\
\dot{w}_{i}=f\left(w_{i}, t\right), \quad i=1,2,3,4 \\
\dot{\delta}_{i}=g\left(\delta_{i}, t\right), \quad i=1,2,3,4 \\
\dot{k}_{i}=-\lambda_{i} e_{i}^{2}, \quad i=1,2,3,4 .
\end{gathered}
$$

The proofs of Theorem 8 follow directly from Theorem 5 . Thus we leave out their proofs here.

Definition 9. For systems (1) and (4), if

$$
\lim _{t \rightarrow \infty}\left\|e_{i}\right\|=\lim _{t \rightarrow \infty}\left\|y_{i}-\left(\int_{0}^{t} \kappa_{i}(s) d s\right) x_{i}\right\|=0
$$

then the status of the response system and the drive system is called the definite integration scaling function projective synchronization (DISFPS).

Remark 10. Similar to Remark $3, \kappa_{i}(t)$ should be bound function; otherwise, one problem should be taken into account if $e_{i}$ is equal to infinite value.

According to Definition 9, similar to Theorems 5 and 8, we may get Theorems 11 and 12 .

Theorem 11. System (1) and system (4) are asymptotically synchronized with the following adaptive control mechanism:

$$
\begin{gathered}
u_{1}=\kappa_{1} x_{1}-y_{1} y_{2}+\left(\int_{0}^{t} \kappa_{1}(s) d s\right) x_{1} x_{2}+k_{1} e_{1}, \\
u_{2}=\kappa_{2} x_{2}+\int_{0}^{t} \kappa_{2}(s) d s-1+y_{1}^{2} \\
-\left(\int_{0}^{t} \kappa_{2}(s) d s\right) x_{1}^{2}+k_{2} e_{2}, \\
u_{3}=\kappa_{3} x_{3}-h v+k_{3} e_{3}, \\
\dot{v}=-1.2 y_{1} y_{2}-0.5 v, \\
\dot{k}_{i}=-\lambda_{i} e_{i}^{2}, \quad i=1,2,3 .
\end{gathered}
$$

Theorem 12. System (1) and system (15) are asymptotically synchronized with the following adaptive control mechanism:

$$
\begin{gathered}
u_{1}=\kappa_{1} x_{1}-y_{1} y_{2}+\left(\int_{0}^{t} \kappa_{1}(s) d s\right) x_{1} x_{2}+k_{1} e_{1}, \\
u_{2}=\kappa_{2} x_{2}+\int_{0}^{t} \kappa_{2}(s) d s-1 \\
+y_{1}^{2}-\left(\int_{0}^{t} \kappa_{2}(s) d s\right) x_{1}^{2}+k_{2} e_{2}, \\
u_{3}=\kappa_{3} x_{3}-h v+k_{3} e_{3}, \\
u_{4}=\kappa_{4} x_{4}+1.2 y_{1} y_{2}+0.5 v+k_{4} e_{4}, \\
\dot{k}_{i}=-\lambda_{i} e_{i}^{2}, \quad i=1,2,3,4 .
\end{gathered}
$$

The proofs of Theorems 11 and 12 follow directly from Theorem 5. Thus we leave out their proofs here.

\section{Simulation and Results}

In this section, computer simulations are used to verify and demonstrate the effectiveness of Theorem 5 .

The dynamical systems $\dot{w}_{i}=f\left(w_{i}, t\right)$ and $\dot{\delta}_{i}=g\left(\delta_{i}, t\right)$ are the following chaotic systems, and two chaotic systems are bounded:

$$
\begin{aligned}
& \dot{w}_{1}=w_{2} w_{3}-2 w_{1}, \\
& \dot{w}_{2}=\left(w_{3}-5\right) w_{1}-2 w_{2}, \\
& \dot{w}_{3}=1-w_{1} w_{2}, \\
& \dot{\delta}_{1}=36\left(\delta_{2}-\delta_{1}\right), \\
& \dot{\delta}_{2}=20 \delta_{2}-\delta_{1} \delta_{3}, \\
& \dot{\delta}_{3}=-3 \delta_{3}+\delta_{1} \delta_{2} .
\end{aligned}
$$

In the simulations, the initial values of the drive and response systems are taken as $(3,1,2)$ and $(-2,3,-3)$, respectively, the initial values of the dynamical systems $\dot{w}_{i}=f\left(w_{i}, t\right)$ and $\dot{\delta}_{i}=g\left(\delta_{i}, t\right)$ are taken as $(6,7,1)$ and $(5,3,7)$ and the initial values of external perturbation system $v=2$ and $k_{i}=1, i=1,2,3$, respectively. The simulated results about the function project synchronization are shown in Figures 1 and 2. In Figure 8, three state errors versus time are shown and it is also shown that the state errors tend to zero asymptotically as time evolves. Figure 9 shows the feedback gain $k_{i}, i=1,2,3$.

Numerical simulations of Theorems 8,11 , and 12 can be illustrated in a similar way as shown in Theorem 5; thus we leave out numerical simulations here.

\section{Conclusion}

First, we have discussed that the three-dimensional chaotic system with external perturbation can be seen as the fourdimensional chaotic system without external perturbation. Second, the definite integral scaling function synchronization 

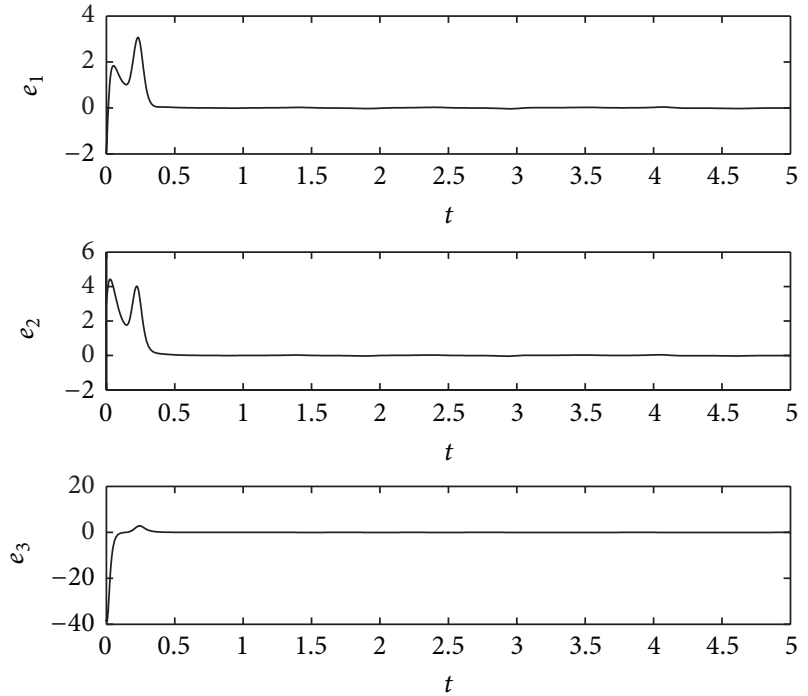

FIGURE 8: Synchronization errors with time $t$.
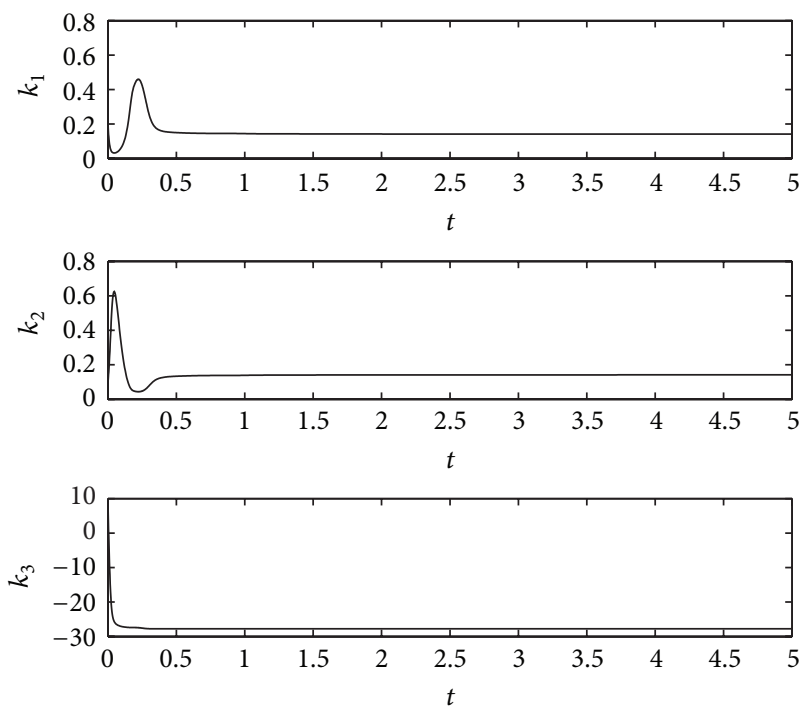

Figure 9: Dynamic curve of the feedback gain $k_{i}, i=1,2,3$.

problem of financial chaotic systems is investigated. Finally, numerical simulations are then given to verify the effectiveness of the proposed adaptive schemes.

\section{Conflict of Interests}

The authors declare that there is no conflict of interests regarding the publication of this paper.

\section{Acknowledgments}

The authors are grateful to the anonymous reviewers and the editor for their valuable comments and suggestions, which have led to a better presentation of this paper. This research is supported by the Youth Fund Project of the Humanities and Social Science Research for the Ministry of Education of China (14YJCZH173), the Science and Technology Research Youth Project for the Education Department of Hubei Province of China (Q20145001), the Science and Technology Research Key Program for the Education Department of Hubei Province of China (D20155001, D20156001), the Natural Science Foundation of Shanghai (15ZR1419000).

\section{References}

[1] J. Ma, Q. Zhang, and Q. Gao, "Stability of a three-species symbiosis model with delays," Nonlinear Dynamics, vol. 67, no. 1, pp. 567-572, 2012.

[2] R. Wang, X. Hui, and X. Zhang, "Analysis of multiple structural changes in financial contagion based on the largest lyapunov exponents," Mathematical Problems in Engineering, vol. 2014, Article ID 209470, 7 pages, 2014.

[3] J. Zheng and B. Du, "Projective synchronization of hyperchaotic financial systems," Discrete Dynamics in Nature and Society, vol. 2015, Article ID 782630, 10 pages, 2015.

[4] C. Xie, Y. Xu, and D. Tong, "Mean square synchronization of stochastic nonlinear delayed coupled complex networks," Discrete Dynamics in Nature and Society, vol. 2013, Article ID 914140, 10 pages, 2013.

[5] M. Salah Abd-Elouahab, N. E. Hamri, and J. Wang, "Chaos control of a fractional-order financial system," Mathematical Problems in Engineering, vol. 2010, Article ID 270646, 18 pages, 2010.

[6] J. Ma, Q. Zhang, and Q. Gao, "Stability of a three-species symbiosis model with delays," Nonlinear Dynamics, vol. 67, no. 1, pp. 567-572, 2012.

[7] R. H. Strotz, J. C. McAnulty, and J. B. Naines, "Goodwin's nonlinear theory of the business cycle: an electro-analog solution," Econometrica, vol. 21, no. 3, pp. 390-411, 1953.

[8] H.-W. Lorenz, Nonlinear Dynamical Economics and Chaotic Motion, Springer, Berlin, Germany, 1993.

[9] K. Sasakura, "On the dynamic behavior of Schinasi's business cycle model," Journal of Macroeconomics, vol. 16, no. 3, pp. 423444, 1994.

[10] L. De Cesare and M. Sportelli, "A dynamic IS-LM model with delayed taxation revenues," Chaos, Solitons \& Fractals, vol. 25, no. 1, pp. 233-244, 2005.

[11] L. Fanti and P. Manfredi, "Chaotic business cycles and fiscal policy: an IS-LM model with distributed tax collection lags," Chaos, Solitons \& Fractals, vol. 32, no. 2, pp. 736-744, 2007.

[12] H. Yu, G. Cai, and Y. Li, "Dynamic analysis and control of a new hyperchaotic finance system," Nonlinear Dynamics, vol. 67, no. 3, pp. 2171-2182, 2012.

[13] Y. Xu, B. Li, Y. Wang, W. Zhou, and J. Fang, "A new fourscroll chaotic attractor consisted of two-scroll transient chaotic and two-scroll ultimate chaotic," Mathematical Problems in Engineering, vol. 2012, Article ID 438328, 12 pages, 2012.

[14] J. H. Ma and Y. S. Chen, "Study for the bifurcation topological structure and the global complicated character of a kind of nonlinear finance system," Applied Mathematics and Mechanics, vol. 22, no. 11, pp. 1119-1128, 2001.

[15] C. C. Wang and C. C. Liu, "Chaotic dynamic analysis of aquatic phytoplankton system," Mathematical Problems in Engineering, vol. 2014, Article ID 586262, 8 pages, 2014. 
[16] Q. L. Zhang and L. Lü, "Synchronization of spatiotemporal chaos in a class of complex dynamical networks," Chinese Physics B, vol. 20, no. 1, Article ID 010510, 2011.

[17] Q. Zhang, Y. Zhang, T. Tanaka, and X. Yan, "Bounded real lemmas for positive descriptor systems," Journal of the Franklin Institute. Engineering and Applied Mathematics, vol. 352, no. 1, pp. 346-368, 2015.

[18] M. Zhao and J. Wang, " $H_{\infty}$ control of a chaotic finance system in the presence of external disturbance and input time-delay," Applied Mathematics and Computation, vol. 233, pp. 320-327, 2014.

[19] Y. Xu, Y. Wang, W. Zhou, and J. Fang, "Stochastic complex networks synchronize to the limit set with adaptive controller and adaptive delay," Mathematical Methods in the Applied Sciences, vol. 37, no. 15, pp. 2290-2296, 2014.

[20] Y. Xu, C. Xie, and Q. Xia, "A kind of binary scaling function projective lag synchronization of chaotic systems with stochastic perturbation," Nonlinear Dynamics, vol. 77, no. 3, pp. 891-897, 2014.

[21] L. Pan and J. Cao, "Stochastic quasi-synchronization for delayed dynamical networks via intermittent control," Communications in Nonlinear Science and Numerical Simulation, vol. 17, no. 3, pp. 1332-1343, 2012.

[22] Y. Tang and W. K. Wong, "Distributed synchronization of coupled neural networks via randomly occurring control," IEEE Transactions on Neural Networks and Learning Systems, vol. 24, no. 3, pp. 435-447, 2013.

[23] Y. Tang, H. Gao, W. Zou, and J. Kurths, "Distributed synchronization in networks of agent systems with nonlinearities and random switchings," IEEE Transactions on Cybernetics, vol. 43, no. 1, pp. 358-370, 2013.

[24] J. Zhao, "Adaptive Q-S synchronization between coupled chaotic systems with stochastic perturbation and delay," Applied Mathematical Modelling, vol. 36, no. 7, pp. 3312-3319, 2012.

[25] R. Zhang, Y. Yang, Z. Xu, and M. Hu, "Function projective synchronization in drive-response dynamical network," Physics Letters A, vol. 374, no. 30, pp. 3025-3028, 2010.

[26] X.-J. Wu and H.-T. Lu, "Adaptive generalized function projective lag synchronization of different chaotic systems with fully uncertain parameters," Chaos, Solitons and Fractals, vol. 44, no. 10, pp. 802-810, 2011.

[27] H. Du, Q. Zeng, C. Wang, and M. Ling, "Function projective synchronization in coupled chaotic systems," Nonlinear Analysis: Real World Applications, vol. 11, no. 2, pp. 705-712, 2010.

[28] Y. Yu and H. X. Li, "Adaptive generalized function projective synchronization of uncertain chaotic systems," Nonlinear Analysis: Real World Applications, vol. 11, no. 4, pp. 2456-2464, 2010.

[29] W. Yang and J. Sun, "Function projective synchronization of two-cell quantum-CNN chaotic oscillators by nonlinear adaptive controller," Physics Letters A, vol. 374, no. 4, pp. 557561,2010 .

[30] H. Du, Q. Zeng, and C. Wang, "Function projective synchronization of different chaotic systems with uncertain parameters," Physics Letters A, vol. 372, no. 33, pp. 5402-5410, 2008.

[31] C.-M. Chang and H.-K. Chen, "Chaos and hybrid projective synchronization of commensurate and incommensurate fractional-order Chen-Lee systems," Nonlinear Dynamics, vol. 62, no. 4, pp. 851-858, 2010.

[32] D. Ghosh and S. Banerjee, "Projective synchronization of timevarying delayed neural network with adaptive scaling factors," Chaos, Solitons \& Fractals, vol. 53, pp. 1-9, 2013.
[33] Z. Wu and X. Fu, "Cluster projective synchronization between community networks with nonidentical nodes," Physica A: Statistical Mechanics and Its Applications, vol. 391, no. 23, pp. 6190-6198, 2012. 


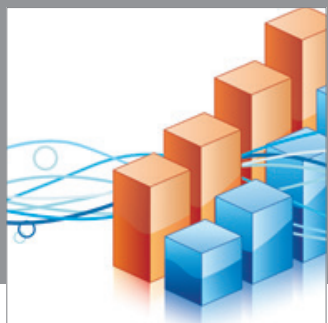

Advances in

Operations Research

mansans

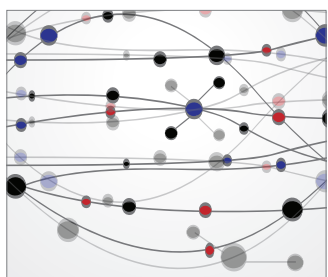

The Scientific World Journal
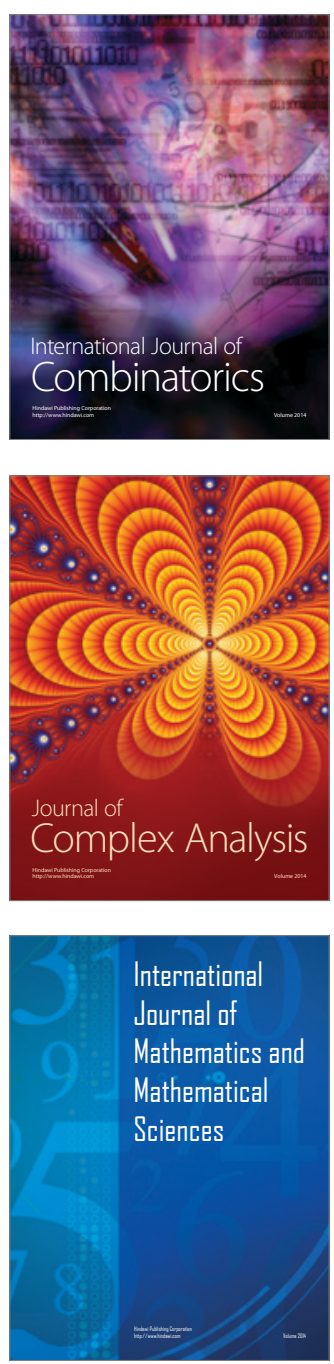
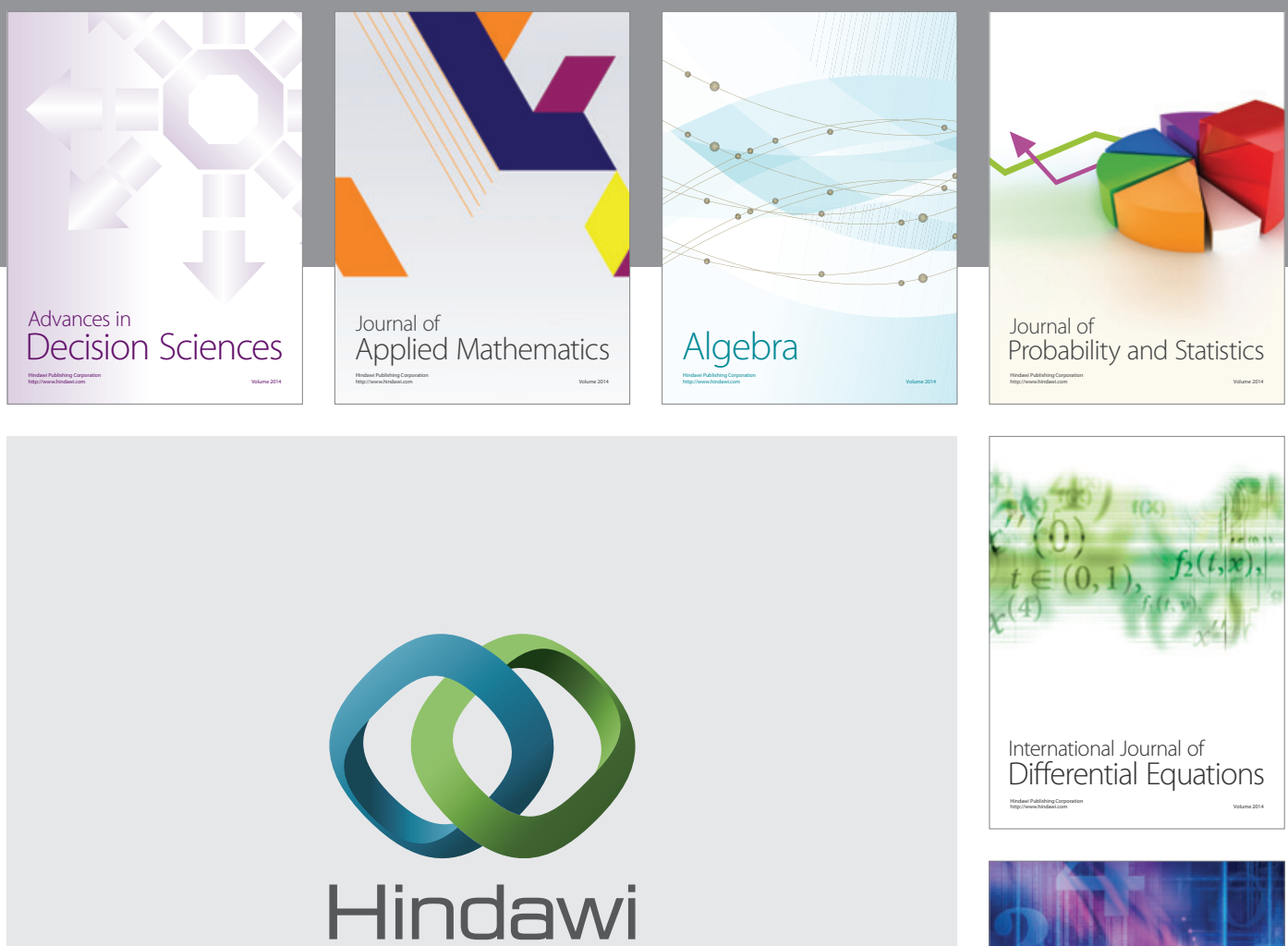

Submit your manuscripts at http://www.hindawi.com
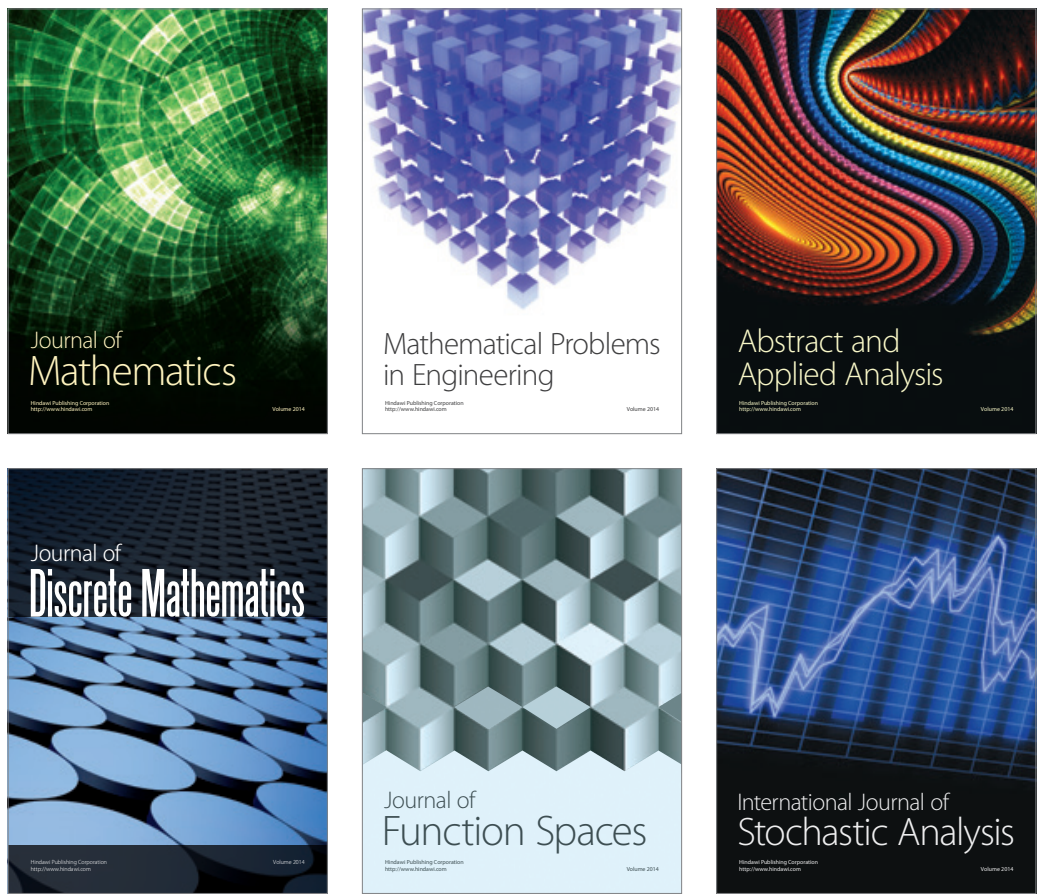

Journal of

Function Spaces

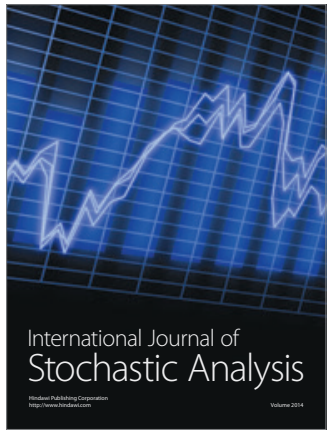

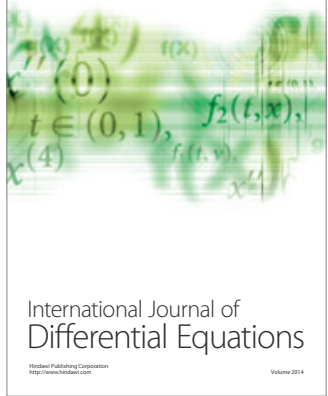
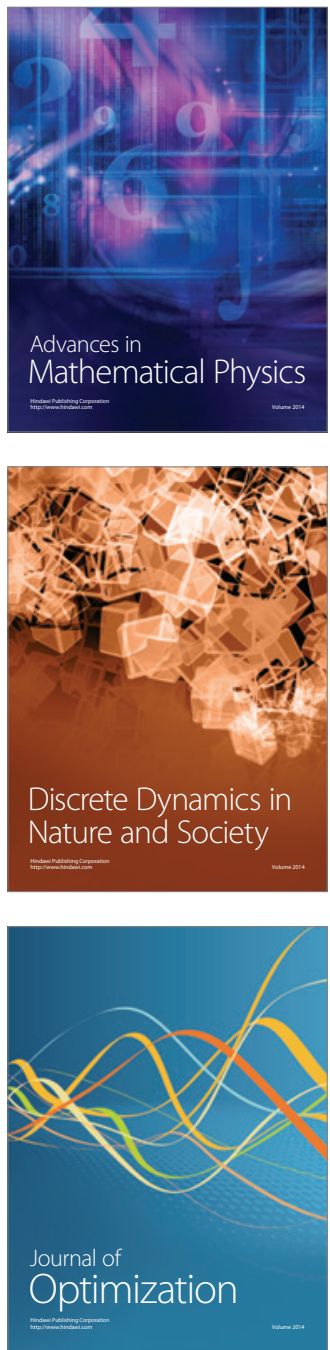